\title{
Odd Lindley-Lomax Model: Statistical Properties and Applications
}

\author{
M. Masoom Ali \\ Department of Mathematical Sciences, Ball State University \\ Muncie, Indiana 47306 USA \\ mali@bsu.edu \\ Mustafa Ç. Korkmaz \\ Deparment of Measurement and Evaluation, Artvin Çoruh University \\ City Campus, Artvin 08000 Turkey \\ mcagatay@artvin.edu.tr ; mustafacagataykorkmaz@gmail.com \\ Haitham M. Yousof \\ Department of Statistics, Mathematics and Insurance \\ Benha University, Egypt \\ haitham.yousof@fcom.bu.edu.eg \\ Nadeem Shafique Butt \\ Department of Family and Community Medicine King Abdul Aziz University \\ Jeddah, Kingdom of Saudi Arabia \\ nshafique@kau.edu.sa
}

\begin{abstract}
In this work, we focus on some new theoretical and computational aspects of the Odd LindleyLomax model. The maximum likelihood estimation method is used to estimate the model parameters. We show empirically the importance and flexibility of the new model in modeling two types of aircraft windshield lifetime data. This model is much better than exponentiated Lomax, gamma Lomax, beta Lomax and other Lomax models so that the Odd Lindley-Lomax lifetime model is a good alternative to these models in modeling aircraft windshield data. A Monte Carlo simulation study is used to assess the performance of the maximum likelihood estimators.
\end{abstract}

Keywords: Lomax model; Odd Lindley-G Family; Estimation.

\section{Introduction}

In the statistical literature, the Lomax (Lo) or Pareto II model was originally pioneered for modeling business failure data by Lomax (1954), and is related to the four-parameter type II generalized beta distribution and the three-parameter Singh-Maddala distribution, as well as the beta distribution of the second kind. The Lo distribution has found a wide application in many fields such as size of cities, income and wealth inequality, actuarial science, engineering, medical and biological sciences, reliability and lifetime modeling. It has been applied to model data obtained from income and wealth (see Harris 1968 and Atkinson and Harrison 1978), firm size (see Corbellini et al., 2007), reliability and life testing (see Hassan Al-Ghamdi 2009), Hirsch-related statistics (see Glanzel 2008), for modeling gauge lengths data (see Afify et al., 2015), for modeling bladder cancer patients data and remission times data (see Yousof et al., 2016 and Yousof et al., 2018), Hamedani et al. (2018) used the type II general exponential Lomax model for modeling failure times of aircraft windshield data, Yousof et al., 2019 introduced a new version of 
the Poisson Lomax distribution, Gad et al., 2019 introduced the Burr XII- Lomax distribution and Elsayed and Yousof 2019 proposed a new Lomax distribution for modeling survival times and taxes revenue data sets.

A random variable (rv) $X$ has the Lo distribution with two parameters $\lambda$ and $\beta$ if it has cumulative distribution function (cdf) (for $x>0$ ) given by

$$
G_{L o}(x ; \lambda, \beta)=1-(1+x / \beta)^{-\lambda},
$$

where $\lambda>0$ and $\beta>0$ are the shape and scale parameters, respectively. Then the corresponding pdf of (1) is

$$
g_{L o}(x ; \lambda, \beta)=\lambda \beta^{-1}(1+x / \beta)^{-(\lambda+1)} .
$$

The goal of this article is to study the Odd Lindley-Lomax (OLLo) model first introduced by Silva et al. (2017) using the Odd Lindley-G (OL-G) family of distributions. The pdf and cdf of the OL-G family of distribution are respectively given by

$$
f(x ; a, \underline{\mathbf{x}})=\left[a^{2} /(1+a)\right] g(x ; \underline{\mathbf{x}}) \bar{G}(x ; \underline{\mathbf{x}})^{-3} \exp [-a G(x ; \underline{\mathbf{x}}) / \bar{G}(x ; \underline{\mathbf{x}})],
$$

and

$$
F(x ; a, \underline{\mathbf{x}})=1-\{[a+\bar{G}(x ; \underline{\mathbf{x}})] /(1+a) \bar{G}(x ; \underline{\mathbf{x}})\} \exp [-a G(x ; \underline{\mathbf{x}}) / \bar{G}(x ; \underline{\mathbf{x}})],
$$

where $a>0$ is the scale parameter, $G(x, \underline{\mathbf{x}})$ is the baseline cdf, $\underline{\mathbf{x}}=(\lambda, \beta)$ is the parameter vector of the baseline distribution and $\bar{G}(x, \underline{\mathbf{x}})=1-G(x, \underline{\mathbf{x}})$ is the survival function of the baseline distribution. To this end, we use equations (1), (2) and (3) to obtain the three-parameter OLLo pdf (for $x>0$ )

$$
f(x ; a, \lambda, \beta)=a^{2} \lambda[\beta(1+a)]^{-1}(1+x / \beta)^{2 \lambda-1} \exp \left\{-a\left[(1+x / \beta)^{\lambda}-1\right]\right\},
$$

From Equation (4), the corresponding cdf of the pdf in (5) is given by

$$
F(x ; a, \underline{\mathbf{x}})=1-\left\{a+(1+x / \beta)^{-\lambda} /\left[(1+a)(1+x / \beta)^{-\lambda}\right]\right\} \exp \left\{-a\left[(1+x / \beta)^{\lambda}-1\right]\right\} .
$$

The hazard rate function (hrf) of the OLLo distribution can be obtained using $\frac{f(x ; a, \lambda, \beta)}{1-F(x ; a, \lambda, \beta)}$. It should be noted that Silva et al. (2017) derived the argument

$$
G(x, \underline{\mathbf{x}}) /[1-G(x, \underline{\mathbf{x}})]=(1+x / \beta)^{\lambda}-1
$$

for the Lomax. We draw the pdf and hrf plots of the OLLo distribution in Figurel for the selected parameters values. We see that its pdf's are unimodal and decreasing shaped. Also, its hrf can be decreasing and increasing shaped. 

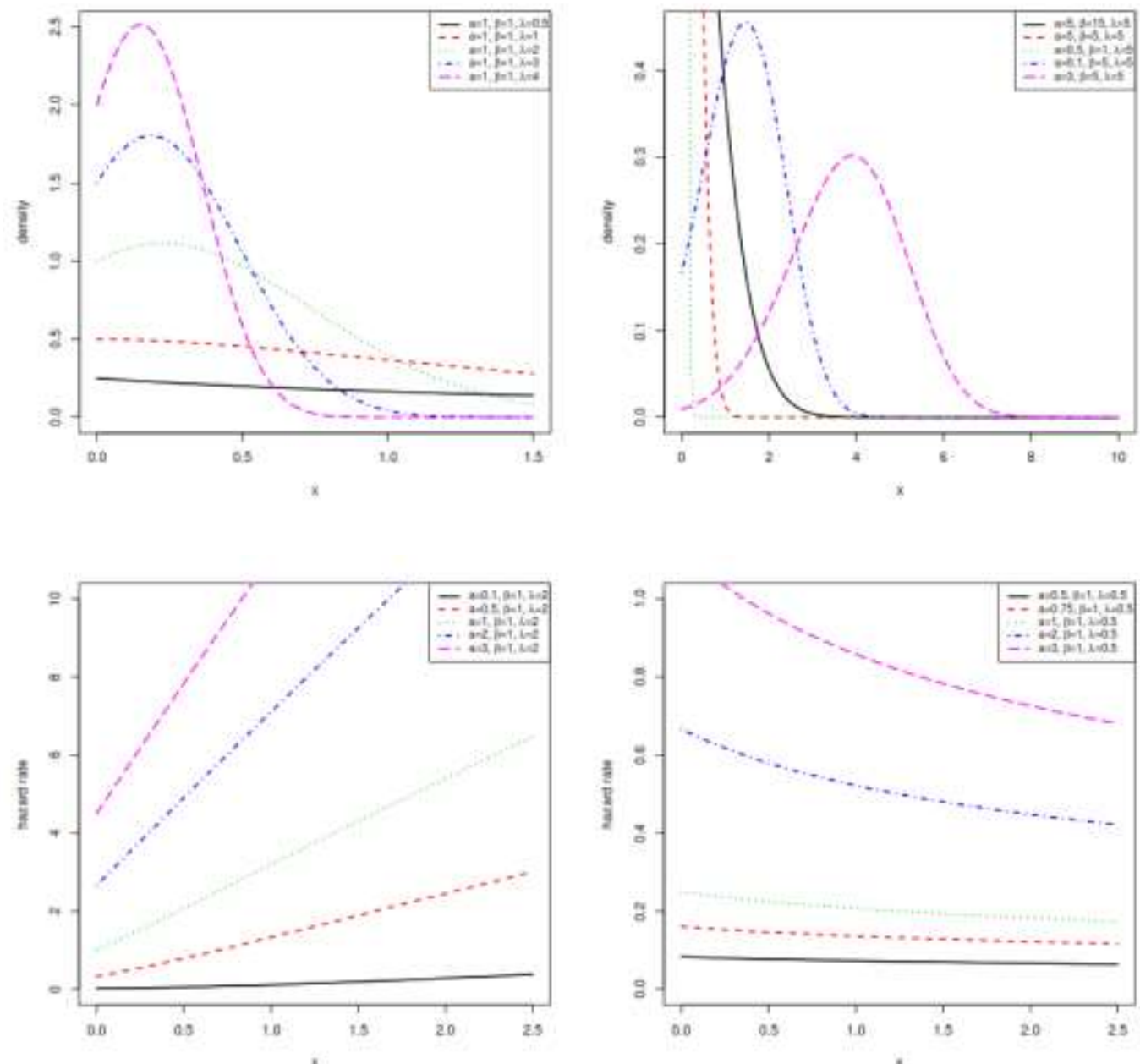

Figure 1: The possible plots for selected OLLo distributions

The OLLo density function can be expressed as an infinite mixture of exponentiated-Lo (exp-Lo) density functions

$$
f(x)=\sum_{m, k=0}^{\infty} \psi_{m, k} \pi_{m+k+1, \lambda, \beta}(x),
$$

where

$$
\psi_{m, k}=(-1)^{k} a^{2+k} \Gamma(m+k+3) /[m ! k !(\lambda+1)(m+k+1) \Gamma(k+3)]
$$

and

$$
g_{m+k+1, \lambda, \beta}(x)=(m+k+1) \underbrace{\lambda \beta^{-1}(1+x / \beta)^{-(\lambda+1)}}_{{ }^{g}{ }_{L o}(x ; \lambda, \beta)} \underbrace{\left[1-(1+x / \beta)^{-\lambda}\right]^{m+k}}_{\left[{ }_{L o}(x ; \lambda, \beta)\right]^{m+k}}
$$

is the exponentiated-Lo (Exp-Lo) density. Similarly,

$$
F(x)=\sum_{k=0}^{\infty} \psi_{m, k} \Pi_{m+k+1, \lambda, \beta}(x)
$$

where 


$$
\Pi_{m+k+1, \lambda, \beta}(x)=\underbrace{\left[1-(1+x / \beta)^{-\lambda}\right]^{m+k}}_{\left[{ }_{L o}(x ; \lambda, \beta)\right]^{m+k+1}}
$$

is the Exp-Lo cdf. In this work, we focus on some new theoretical and computational aspects of the OLLo model.

The rest of the paper is outlined as follows. In Section 2, we derive some of its statistical properties including moments, generating function, residual life and reversed residual life functions and order statistics and their moments are introduced at the end of the section. Maximum likelihood estimation of the model parameters is addressed in Section 3. In Section 4, simulation results to assess the performance of the proposed maximum likelihood estimations, we provide the applications to real data sets to illustrate the importance of the new family in Section 5. Finally, we offer some concluding remarks in Section 6.

\section{Statistical properties}

In this section, we provide some mathematical properties of the OLLo distribution. The formulas derived in this section are manageable and simple, and with the use of advanced computer resources and their numerical computing capabilities, the OLLo model may prove to be a useful addition to those distributions which are used for modeling data in reliability, economics, medicine engineering, among others.

\subsection{Moments and cumulants}

The various types of moments of a rv are important especially in applied areas. Many of the most important features and characteristics of a certain distribution can be studied through moments, e.g., tendency, dispersion, skewness and kurtosis etc. The $r^{\text {th }}$ ordinary moment of $X$ is given by

$$
\mu_{r}^{\prime}=E\left(X^{r}\right)=\sum_{m, k=0}^{\infty} \psi_{m, k} \int_{0}^{\infty} x^{r} \pi_{m+k+1, \lambda, \beta}(x) d x,
$$

then we obtain

$$
\mu_{r}^{\prime}=\sum_{m, k=0}^{\infty} \sum_{w=0}^{r} \psi_{m, k, w}^{(r, m+k+1)} \mathbf{B}(m+k+1,1+(w-r) / \lambda), \forall r \leq \lambda,
$$

where

$$
\mathbf{B}(p ; q)=\int_{0}^{1} u^{p-1}(1-u)^{q-1} d u
$$

is the complete beta function and

$$
\psi_{m, k, w}^{(r, m+k+1)}=\psi_{m, k}(m+k+1) \beta^{r}(-1)^{w}\left(\begin{array}{l}
r \\
w
\end{array}\right) .
$$

Setting $r=1$ in (9), we have the mean of $X$. The last integration can be computed numerically for most parent distributions. The skewness and kurtosis measures can be calculated from the ordinary moments using well-known relationships. The $r^{\text {th }}$ central moment of $X$, say $M_{r}$, is 


$$
\mu_{r}=E(X-\mu)^{r}=\sum_{h=0}^{r}(-1)^{h}\left(\begin{array}{l}
r \\
h
\end{array}\right)\left(\mu_{1}^{\prime}\right)^{r} \mu_{r-h}^{\prime},
$$

where $\mu=E(X)$. The skewness and kurtosis measures also can be calculated from the ordinary moments using well-known relationships. For the skewness and kurtosis coefficients, we have

$$
\sqrt{\beta_{1}}=\sqrt{\frac{\mu_{3}^{2}}{\mu_{2}^{3}}} \text { and } \beta_{2}=\frac{\mu_{4}}{\mu_{2}^{2}}
$$

respectively. The cumulants $\left(\kappa_{n}\right)$ of $X$ follow recursively from $\kappa_{n}=\mu_{n}^{\prime}-\sum_{r=0}^{n-1}\left(\begin{array}{c}n-1 \\ r-1\end{array}\right) \kappa_{r} \mu_{n-r}^{\prime}$, where $\kappa_{1}=\mu_{1}^{\prime}, \kappa_{2}=\mu_{2}^{\prime}-\mu_{1}^{\prime 2}, \kappa_{3}=\mu_{3}^{\prime}-3 \mu_{2}^{\prime} \mu_{1}^{\prime}+\mu_{1}^{3}$, and so on. The values for mean, variance, $\sqrt{\beta_{1}}$ and $\beta_{2}$ for selected values of $a, \lambda$ and $\beta$ are shown in Table1. We can say that the OLLo model can be useful for various data modelling in terms of skewness and kurtosis.

Table 1: Mean, variance, coefficients of skewness and kurtosis for different values of parameters

\begin{tabular}{lllll}
\hline \multicolumn{1}{c}{$(a, \lambda, \beta)$} & $\mu$ & $\operatorname{Var}(X)$ & $\sqrt{\beta_{1}}$ & $\beta_{2}$ \\
\hline$(0.5,0.5,0.5)$ & 12.6666 & 354.2228 & 4.2064 & 35.9142 \\
$(1,0.5,0.5)$ & 3.5000 & 24.7500 & 4.0221 & 33.0892 \\
$(2,0.5,0.5)$ & 1.0833 & 2.0347 & 3.6468 & 27.6014 \\
$(0.5,1,0.5)$ & 1.6666 & 1.8888 & 1.5122 & 6.3425 \\
$(0.5,2,0.5)$ & 0.4945 & 0.0943 & 0.6863 & 3.2650 \\
$(0.5,0.5,1)$ & 25.3333 & 1416.8910 & 4.2065 & 35.9142 \\
$(0.5,0.5,2)$ & 50.6666 & 5667.5550 & 4.2064 & 35.9143 \\
$(0.05,5,1)$ & 1.0072 & 0.0921 & -0.2019 & 2.9039 \\
$(0.1,5,1)$ & 0.7502 & 0.0678 & -0.1422 & 2.7800 \\
$(0.2,5,1)$ & 0.5311 & 0.0476 & -0.0216 & 2.6218 \\
\hline
\end{tabular}

The mean, variance, skewness and kurtosis of the OLLo distribution are computed numerically for different values of parameters using the $\mathrm{R}$ software. The numerical values displayed in Table 1 indicate that the skewness of the OLLo distribution can range in the interval $(-0.023,4.23)$. The spread for its kurtosis is much larger ranging from 2.6 to 36 .

\subsection{Generating function}

The moment generating function (mgf) $M_{X}(t)=E\left(e^{t X}\right)$ can be derived from equation (7) as follows

$$
M_{X}(t)=\sum_{w, k, r=0}^{\infty} \sum_{w=0}^{r} \frac{t^{r}}{r !} \psi_{m, k, w}^{(r, m+k+1)} \mathbf{B}(m+k+1,1+(w-r) / \lambda), \forall r \leq \lambda,
$$

\subsection{Incomplete moments and mean deviations}

The main applications of the first incomplete moment refer to the mean deviations and the Bonferroni and Lorenz curves. These curves are very useful in economics, reliability, 
demography, insurance and medicine. The $s^{\text {th }}$ incomplete moment, say $I_{s}(t)$, of $X$ can be expressed from (7) as

$$
I_{s}(t)=\sum_{m, k=0}^{\infty} \sum_{w=0}^{s} \psi_{m, k, w}^{(s, m+k+1)} \mathbf{B}_{t}(m+k+1,1+(w-s) / \lambda), \forall s \leq \lambda .
$$

where

$$
\mathbf{B}_{t}(p ; q)=\int_{0}^{t} u^{p-1}(1-u)^{q-1} d u
$$

is the incomplete beta function. The mean deviations about the mean $\left[\delta_{1}=E(|X-E(X)|)\right]$ and about the median $\left[\delta_{2}=E(|X-\operatorname{Median}(X)|)\right]$ of $X$ are given by $\delta_{1}=2 \mu_{1}^{\prime} F\left(\mu_{1}^{\prime}\right)-2 I_{1}\left(\mu_{1}^{\prime}\right)$ and $\delta_{2}=\mu_{1}^{\prime}-2 I_{1}(M)$, respectively, where $\operatorname{Median}(X)=Q(0.5)$ is the median, $F\left(\mu_{1}^{\prime}\right)$ is easily calculated from (5) and $I_{1}(t)$ is the first incomplete moment given by the last Equation with $s=1$. A general equation for $I_{1}(t)$ can be derived from $I_{s}(t)$ as

$$
I_{1}(t)=\sum_{m, k=0}^{\infty} \sum_{w=0}^{r} \psi_{m, k, w}^{(1, m+k+1)} \mathbf{B}_{t}(m+k+1,1+(w-1) / \lambda) .
$$

\subsection{Moment of residual and reversed residual life}

The $n^{t h}$ moment of the residual life, say $m_{n}(t)=E\left[(X-t)^{n} \mid X>t\right], n=1,2, \ldots$, uniquely determines $F(x)$. The $n^{\text {th }}$ moment of the residual life of $X$ is given by

therefore,

$$
m_{n}(t)=[1-F(t)]^{-1} \int_{t}^{\infty}(x-t)^{n} d F(x)
$$

$$
m_{n}(t)=[1-F(t)]^{-1} \sum_{m, k=0}^{\infty} \sum_{w=0}^{s} \psi_{m, k, w}^{(n, m+k+1)^{\star}} \mathbf{B}_{t}(m+k+1,1+(w-n) / \lambda), \forall n \leq \lambda,
$$

where

$$
\psi_{m, k, w}^{(n, m+k+1)^{\star}}=\psi_{m, k, w}^{(n, m+k+1)} \sum_{d=0}^{n}(1-t)^{n} .
$$

Another interesting function is the mean residual life (MRL) function or the life expectation at age $t$ defined by $m_{1}(t)=E[(X-t) \mid X>t]$, which represents the expected additional life length for a unit which is alive at age $t$. The MRL of $X$ can be obtained by setting $n=1$ in the previous equation. The $n^{\text {th }}$ moment of the reversed residual life, say $M_{n}(t)=E\left[(t-X)^{n} \mid X \leq t\right]$ for $t>0$ and $n=1,2, \ldots$ uniquely determines $F(x)$. We obtain

$$
M_{n}(t)=F(t)^{-1} \int_{0}^{t}(t-x)^{n} d F(x) .
$$

Then, the $n^{\text {th }}$ moment of the reversed residual life of $X$ becomes

$$
M_{n}(t)=F(t)^{-1} \sum_{m, k=0}^{\infty} \sum_{w=0}^{s} \psi_{m, k, w}^{(n, m+k+1)^{\star \star}} \mathbf{B}_{t}(m+k+1,1+(w-n) / \lambda), \forall n \leq \lambda,
$$

where 


\subsection{Order statistics}

$$
\psi_{m, k, w}^{(n, m+k+1)^{\star \star}}=\psi_{m, k, w}^{(n, m+k+1)} \sum_{d=0}^{n}(-1)^{d}\left(\begin{array}{l}
n \\
d
\end{array}\right) t^{n-d} .
$$

Let $X_{1}, \ldots, X_{n}$ be a random sample from the OLLo model of distributions and let $X_{1: n}, \ldots, X_{n: n}$ be the corresponding order statistics. The pdf of the $i^{\text {th }}$ order statistic, say $X_{i: n}$, can be expressed as

$$
f_{i: n}(x)=[\mathrm{B}(i, n-i+1)]^{-1} f(x) F(x)^{i-1}[1-F(x)]^{n-i},
$$

where $B(\cdot, \cdot)$ is the beta function. Substituting (5) and (6) in Equation (10), we obtain

$$
f_{i: n}(x)=\sum_{m, p=0}^{\infty} \sum_{j=0}^{k+n-i} \psi_{m, p, j} \pi_{j+m+p, \lambda, \beta}(x),
$$

where

$$
\psi_{m, p, j}=\sum_{k=0}^{i-1} \frac{(-1)^{k+m} \lambda^{j+m+2}(1+\lambda)^{-(j+1)}}{m ! \mathrm{B}(i, n-i+1)(j+m+p+1)}\left(\begin{array}{c}
j+m+p \\
j+m
\end{array}\right)\left(\begin{array}{c}
k+n-1 \\
j
\end{array}\right)\left(\begin{array}{c}
i-1 \\
k
\end{array}\right) .
$$

Then, the $q^{\text {th }}$ moment of $X_{i: n}$ is given by

$$
\begin{array}{r}
E\left(X_{i: n}^{q}\right)=\sum_{m, p=0}^{\infty} \sum_{j=0}^{k+n-i} \sum_{w=0}^{r} \psi_{m, k, j, w}^{(q, m+k+1)} \mathbf{B}(m+k+1,1+(w-q) / \lambda), \forall \lambda>q, \\
\text { where } \\
\psi_{m, k, j, w}^{(q, m+k+1)}=\psi_{m, p, j}(j+m+p) \beta^{q}(-1)^{w}\left(\begin{array}{c}
q \\
w
\end{array}\right) .
\end{array}
$$

\section{Estimation}

Several approaches for parameter estimation has been proposed in the literature but maximum likelihood method is the most commonly employed. The maximum likelihood estimators (MLEs) enjoy desirable properties and can be used for constructing confidence intervals and regions and also in test statistics. The normal approximation for these estimators in large samples can be easily handled either analytically or numerically. So, we consider the estimation of the unknown parameters of this family from complete samples only by maximum likelihood. Let $x_{1}, \ldots, x_{n}$ be a random sample from OLLo distribution with parameters $a, \lambda, \beta$ and $b$. Let $\mathbf{h}=(a, \lambda, \beta)^{\top}$ be the $3 \times 1$ parameter vector. For determining the MLE of $\mathbf{h}$, we have the log-likelihood function

$$
\ell=\ell(\mathbf{h})=2 n \log a-n \log (1+a)+n \log \lambda-n \log \beta+(2 \lambda-1) \sum_{i=1}^{n} \log s_{i}-a \sum_{i=1}^{n}\left(s_{i}^{\lambda}-1\right),
$$

where $s_{i}=(1+x / \beta)$.

The components of the score vector, $U_{h}=\left(\frac{\partial}{\partial a}, \frac{\partial}{\partial \lambda}, \frac{\partial}{\partial \beta}\right)^{\top}$, are

$$
\begin{gathered}
U_{a}=2 n / a-n /(1+a)-\sum_{i=1}^{n}\left(s_{i}^{\lambda}-1\right), U_{\lambda}=n / \lambda+2 \sum_{i=1}^{n} \log s_{i} \\
U_{\lambda}=n / \lambda+2 \sum_{i=1}^{n} \log s_{i},
\end{gathered}
$$

and 


$$
U_{\beta}=-n / \beta-(2 \lambda-1) \beta^{-2} \sum_{i=1}^{n} x_{i} s_{i}^{-1}+a \lambda \beta^{-2} \sum_{i=1}^{n} x_{i} s_{i}^{\lambda-1} .
$$

Equation (12) can be maximized either directly by using the $\mathrm{R}$ (optim function), Ox program (sub-routine MaxBFGS) or SAS (PROC NLMIXED) or by solving the nonlinear likelihood equations obtained by differentiating (12). Setting the nonlinear system of equations $U_{a}=0, U_{\lambda}=0$ and $U_{b}=0$ and solving them simultaneously yields the $\operatorname{MLE} \mathbf{h}=(a, \lambda, \beta)^{\mathrm{T}}$. To solve these equations, it is usually more convenient to use nonlinear optimization methods such as the quasi-Newton algorithm to numerically maximize $\ell$. For interval estimation of the parameters, we obtain the $3 \times 3$ observed information matrix $J(\mathbf{h})=\left\{\frac{\partial^{2} \ell}{\partial r \partial s}\right\}$ (for $r, s=a, \lambda, \beta$ ), whose elements can be computed numerically. Under standard regularity conditions when $n \rightarrow \infty$, the distribution of $\mathbf{h}$ can be approximated by a multivariate normal $N_{3}\left(0, J(\mathbf{h})^{-1}\right)$ distribution to construct approximate confidence intervals for the parameters. Here, $J(\mathbf{h})$ is the total observed information matrix evaluated at $\mathbf{h}$. Further works could be addressed using different methods to estimate the OLLo parameters such as least squares, weighted least squares, moments, bootstrap, Jackknife, Anderson-Darling, Cramer-von-Mises, Bayesian analysis, among others, and compare the estimators based on these methods.

\section{Simulation study}

In this section, we conduct the simulation study to see the performance of the MLEs of the OLLo distribution with respect to sample size n. We generated $N=1000$ samples of size $n=20,30, \ldots, 500$ from OLLo distribution with $a=12, \beta=0.5$ and $\lambda=2$ by using inverse transform method. The MLEs, say $\left(\hat{a}_{i}, \hat{\beta}_{i}, \hat{\lambda}_{i}\right)$ for $=1,2, \ldots, N$, have been obtained by using CG routine in R programme. Further, we calculate emprical mean, standard deviations (sd), bias and mean square error of the estimate (MSE) for MLEs. The empirical bias and MSE are calculated by (for $h=a, \beta, \lambda$ )

$$
\operatorname{Bias}_{h}(n)=\frac{1}{N} \sum_{i=1}^{N}\left(h_{i}-\hat{h}_{i}\right) \text { and } \operatorname{MSE}_{h}(n)=\frac{1}{N} \sum_{i=1}^{N}\left(h_{i}-\hat{h}_{i}\right)^{2} .
$$

The results are presented in Figure2. From Figure2, we can say that for three parameters the emprical means are very close to true parameter values and they are quite stable. Moreover, the bias, MSE and sd decrease as sample size increases. These above results are as expected.
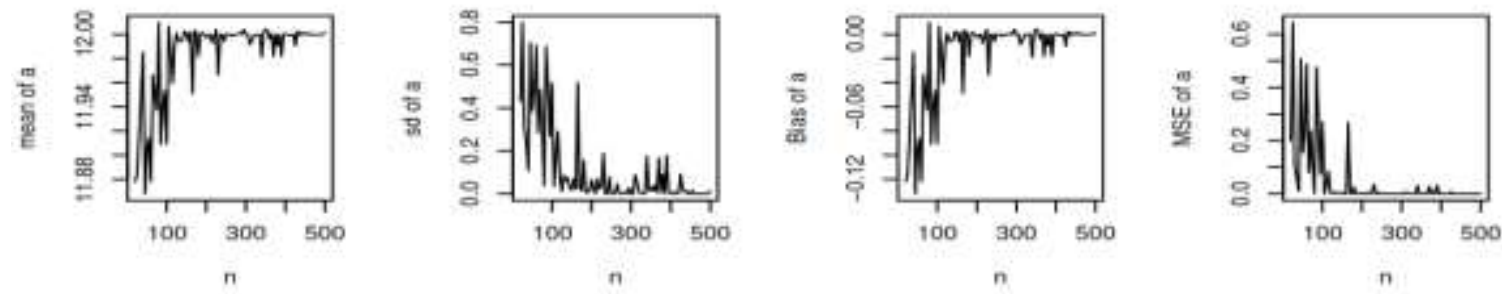

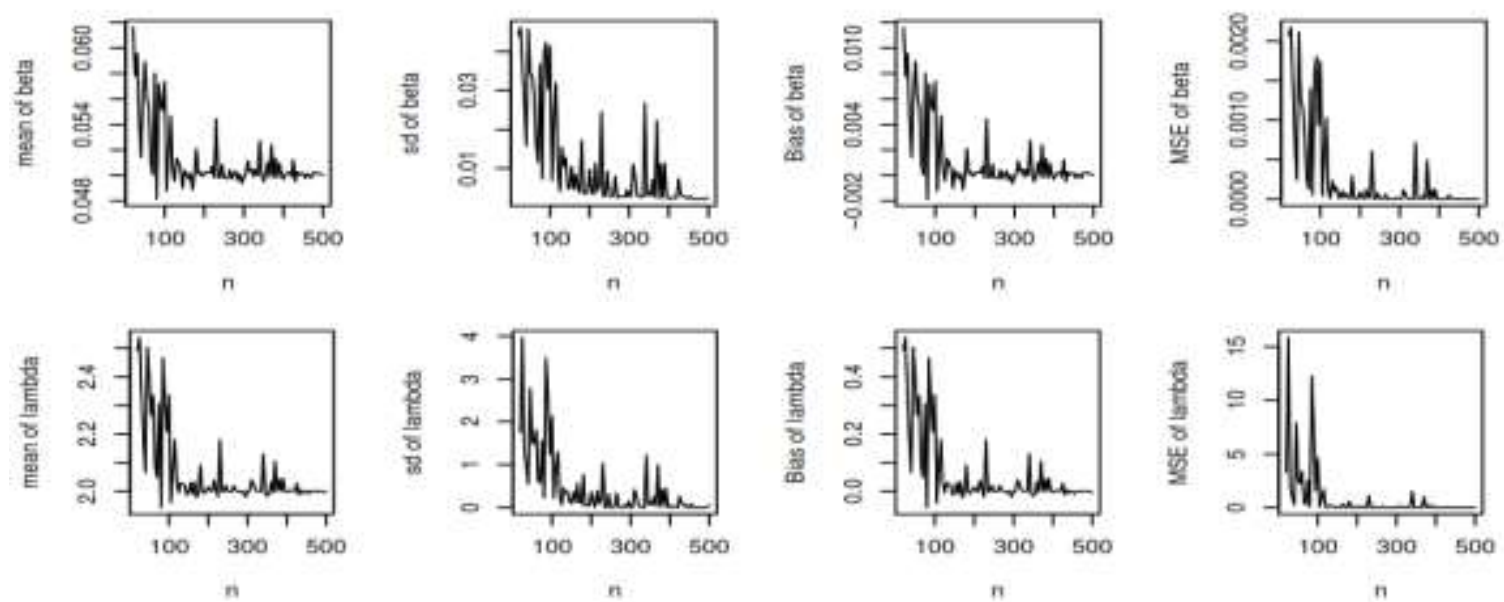

Figure 2: Plots of the emrical mean, sd, biases and MSE of $a, \beta, \lambda$ versus $n$

\section{Applications}

In this section, we provide two applications to two real data sets to prove the importance and flexibility of the OLLo distribution. We compare the fit of the OLLo with competitve models namely: exponentiated Lomax (ELo) model (Gupta et al., 1998), gamma Lomax (KwLo) model (Cordeiro et al., 2015), beta Lomax (BLo) model (Lemonte and Cordeiro, 2013) and Lo model. The cdfs of these distributions are, respectively, given by (for $x>0$ and $\alpha, \beta, \lambda, a>0$ ):

$$
\begin{gathered}
F_{E L o}(x ; \alpha, \beta, \lambda)=\left[1-\left(1+\frac{x}{\beta}\right)^{-\lambda}\right]^{\alpha}, \\
F_{G L o}(x ; \alpha, \beta, \lambda)=\Gamma^{-1}(\alpha) \Gamma\left(\alpha ; \lambda \log \left[1+\frac{x}{\beta}\right]\right)
\end{gathered}
$$

and

$$
F_{B L o}(x ; \alpha, \beta, a, \lambda)=\frac{1}{B(\alpha, a)} B\left(1-\left(1+\frac{x}{\beta}\right)^{-\lambda} ; \alpha, a\right),
$$

where $\Gamma(\cdot)$ is the gamma function, $\Gamma(\cdot ;)$ is the incomplete gamma function, $B(\cdot, \cdot)$ is the complete beta function and $\mathrm{B}(\because ;, \cdot)$ is the incomplete beta function.

The first real data set represents the data on failure times of 84 aircraft windshield given in Murthy et al. (2004). The data are: 0.040, 1.866, 2.385, 3.443, 0.301, 1.876, 2.481, 3.467, 0.309, 1.899, 2.610, 3.478, 0.557, 1.911, 2.625, 3.578, 0.943, 1.912, 2.632, $3.595,1.070,1.914,2.646,3.699,1.124,1.981,2.661,3.779,1.248,2.010,2.688,3.924$, $1.281,2.038,2.82,3,4.035,1.281,2.085,2.890,4.121,1.303,2.089,2.902,4.167,1.432$, 2.097, 2.934, 4.240, 1.480, 2.135, 2.962, 4.255, 1.505, 2.154, 2.964, 4.278, 1.506, 2.190, $3.000,4.305,1.568,2.194,3.103,4.376,1.615,2.223,3.114,4.449,1.619,2.224,3.117$, $4.485,1.652$, 2.229, 3.166, 4.570, 1.652, 2.300, 3.344, 4.602, 1.757, 2.324, 3.376, 4.663.

The second real data set represents the data on service times of 63 aircraft windshield given in Murthy et al. (2004). The data are: 0.046, 1.436, 2.592, 0.140, 1.492, 
$2.600,0.150,1.580,2.670,0.248,1.719,2.717,0.280,1.794,2.819,0.313,1.915,2.820$, $0.389,1.920,2.878,0.487,1.963,2.950,0.622,1.978,3.003,0.900,2.053,3.102,0.952$, $2.065,3.304,0.996,2.117,3.483,1.003,2.137,3.500,1.010,2.141,3.622,1.085,2.163$, $3.665,1.092,2.183,3.695,1.152,2.240,4.015,1.183,2.341,4.628,1.244,2.435,4.806$, $1.249,2.464,4.881,1.262,2.543,5.140$.

These data sets were recently studied by Tahir et al. (2015). The unit for measurement is $1000 \mathrm{~h}$ for both data sets.

In order to compare the distributions, the estimated log-likelihood values $\hat{\ell}$, Akaike Information Criteria (AIC), Cramer von Mises $\left(W^{*}\right)$ and Anderson-Darling $\left(A^{*}\right)$ goodness of-fit statistics were calculated for all models. The statistics $W^{*}$ and $A^{*}$ are described in detail in Chen and Balakrishnan (1995). In general, it can be chosen as the best model which has the smaller values of the AIC, $W^{*}$ and $A^{*}$ statistics and the larger values of $\hat{\ell}$. The required computations are obtained by using the "maxLik" and "goftest" sub-routines in R-software. The analysis results of both these applications are listed in Tables 2 and 3. These results show that the OLLo distribution has the lowest AIC, $W^{*}$ and $A^{*}$ values and has the biggest estimated log-likelihood among all the fitted models. Hence, it could be chosen as the best model under these criteria.

Table 2: MLEs, standard erros of the estimates (in parentheses), $\hat{\ell}$ and goodness-of-fits statistics for the first data

\begin{tabular}{lllllllll}
\hline Model & $\alpha$ & $\beta$ & $a$ & $\lambda$ & $-\hat{\ell}$ & $A I C$ & $A^{*}$ & $W^{*}$ \\
\hline OLLo & & 3.9901 & 0.2147 & 4.1792 & 128.0370 & 262.0740 & 0.5601 & 0.0793 \\
& & $(9.1545)$ & $(0.2293)$ & $(5.4552)$ & & & & \\
BLo & 3.6036 & 118.8374 & 33.6387 & 4.8307 & 138.7177 & & & \\
& $(0.6187)$ & $(63.7145)$ & $(9.2382)$ & $(429.0000)$ & & 285.4354 & 1.4084 & 0.1680 \\
ELo & 3.6261 & 26257.6808 & & 20074.5097 & 141.3997 & & & \\
& $(0.6236)$ & $(99.7417)$ & & $(2041.8263)$ & & 288.7994 & 1.7435 & 0.2194 \\
GLo & 3.5876 & 37029 & & 52001 & 138.4042 & & & \\
& $(0.5133)$ & $(81.1644)$ & & $(7955)$ & & 282.8093 & 1.3667 & 0.1619 \\
Lo & & 131789 & & 51425 & 164.9900 & & & \\
& & $(296.1200)$ & & $(5933.49)$ & & 333.9767 & 1.3976 & 0.1665 \\
\hline
\end{tabular}

Table 3: MLEs, standard erros of the estimates (in parentheses), $\hat{\ell}$ and goodness-of-fits statistics for the second data

\begin{tabular}{|c|c|c|c|c|c|c|c|c|}
\hline Model & $\alpha$ & $\beta$ & $a$ & $\lambda$ & $-\hat{\ell}$ & $A I C$ & $A^{*}$ & $W^{*}$ \\
\hline OLLo & & $\begin{array}{l}9.8798 \\
(4.2235)\end{array}$ & $\begin{array}{c}0.7637 \\
(0.2303)\end{array}$ & $\begin{array}{l}5.2119 \\
(1.7626)\end{array}$ & 98.1289 & 202.2579 & 0.2477 & 0.0351 \\
\hline BLo & $\begin{array}{c}1.9218 \\
(0.3185)\end{array}$ & $\begin{array}{l}169.5800 \\
(339.2068)\end{array}$ & $\begin{array}{l}31.2595 \\
(316.8413)\end{array}$ & $\begin{array}{l}4.9685 \\
(50.5279)\end{array}$ & 102.9611 & 213.9223 & 1.1336 & 0.1872 \\
\hline ELo & $\begin{array}{c}1.9145 \\
(0.3483)\end{array}$ & $\begin{array}{l}32881.9 \\
(162.2230)\end{array}$ & & $\begin{array}{r}22971.2 \\
(3209.5)\end{array}$ & 103.5468 & 213.9223 & 1.2331 & 0.2037 \\
\hline GLo & $\begin{array}{c}1.9073 \\
(0.3214)\end{array}$ & $\begin{array}{l}39197.6 \\
(151.6530)\end{array}$ & & $\begin{array}{c}35842.4 \\
(6945.0)\end{array}$ & 102.8333 & 211.6664 & 1.1121 & 0.2038 \\
\hline Lo & & $\begin{array}{l}207019 \\
(301.2370)\end{array}$ & & $\begin{array}{l}99269 \\
(11863.5222)\end{array}$ & 109. & 222.5976 & 1.1265 & 0.1861 \\
\hline
\end{tabular}


The plots of the fitted densities, cdfs and and probability-probability (P-P) plot of OLLo model are displayed in Figures 3 and 4. We can see that the OLLo distribution provides a good fit and can be used as a competitive model to the other considered models from these Figures.
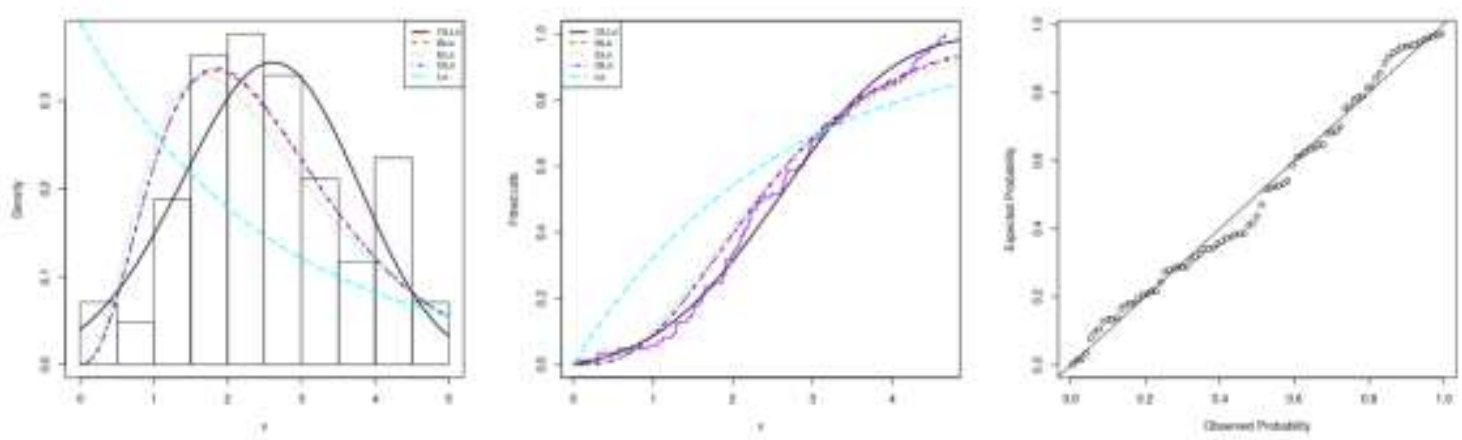

Figure 3: The fitted pdfs (left), cdfs (middle) and P-P plot (right) for the first data set
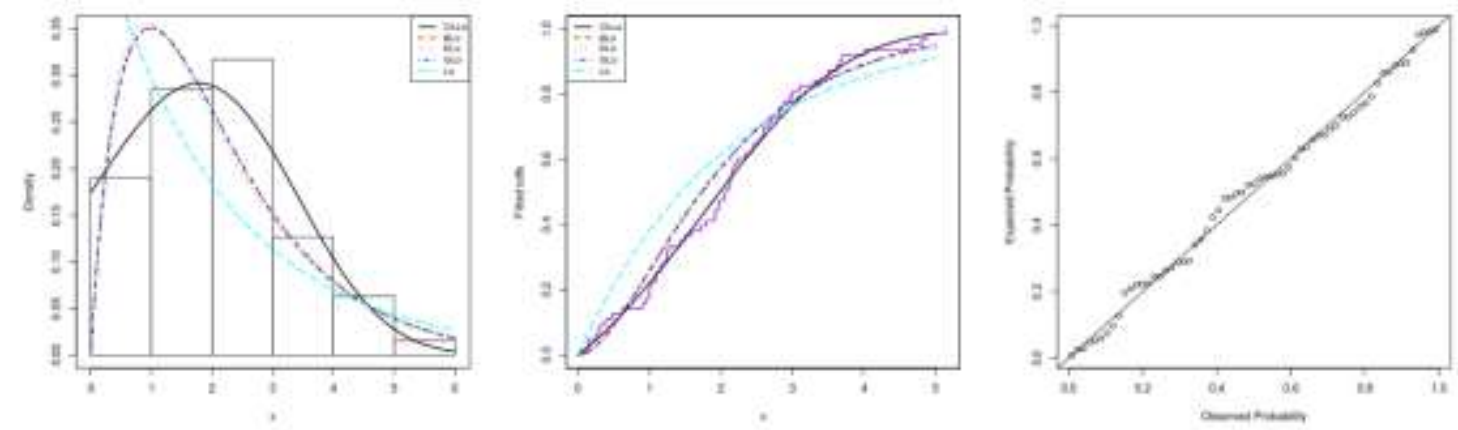

Figure 4: The fitted pdfs (left), cdfs (middle) and P-P plot (right) for the second data set

\section{Conclusions}

In this work, we focus on some new theoretical and computational aspects of the Odd Lindley-Lomax model. A Monte Carlo simulation study is used to assess the performance of the proposed maximum likelihood estimations. The maximum likelihood estimation method is used to estimate the model parameters. We show empirically the importance and flexibility of the new model in modeling two types of aircraft windshield lifetime data. We hope that the new model will attract a wider applications in engineering, reliability, and other areas of research. As a future work we will consider bivariate and multivariate extension of the Odd Lindley-Lomax distribution. In particular with the copula based construction method, trivariate reduction etc.

\section{References}

1. Afify, A. Z., Nofal, Z. M., Yousof, H. M., El Gebaly, Y. M. and Butt, N. S. (2015). The transmuted Weibull Lomax distribution: properties and application. Pak. J. Stat. Oper. Res., 11 (1), 135-152.

2. Atkinson, A.B. and Harrison, A.J. (1978). Distribution of Personal Wealth in Britain (Cambridge University Press, Cambridge). 
3. Chen, G. and Balakrishnan, N. (1995). A general purpose approximate goodness-of-fit test. Journal of Quality Technology, 27, 154-161.

4. Elsayed, H. A. H. and Yousof, H. M. (2019). A new Lomax distribution for modeling survival times and taxes revenue data sets. Journal of Statistics and Applications, forthcoming.

5. Gad, A. M., Hamedani, G. G., Salehabadi, S. M. and Yousof, H. M. (2019). The Burr XII-Burr XII distribution: mathematical properties and characterizations. Pakistan Journal of Statistics, forthcoming.

6. Gupta, R. C., Gupta, P. L. and Gupta, R. D. (1998). Modeling failure time data by Lehman alternatives. Communications in Statistics-Theory and methods, 27(4), 887-904.

7. Corbellini, A., Crosato, L., Ganugi, P and Mazzoli, M. (2007). Fitting Pareto II distributions on firm size: Statistical methodology and economic puzzles. Paper presented at the International Conference on Applied Stochastic Models and Data Analysis, Chania, Crete.

8. Cordeiro, G. M., Ortega, E. M. M. and Popovic, B. V. (2015). The gamma-Lomax distribution. Journal of Statistical computation and Simulation, 85(2), 305-319.

9. Glanzel, W. (2008). On some new bibliometric applications of statistics related to the hindex, Scientometrics 77, 187-196.

10. Hamedani G. G. Rasekhi, M., Najibi, S. M., Yousof, H. M. and Alizadeh, M. (2018). Type II general exponential class of distributions. Pak. J. Stat. Oper. Res., forthcoming.

11. Harris, C. M. (1968). The Pareto distribution as a queue service descipline, Operations Research, 16, 307--313.

12. Hassan, A.S. and Al-Ghamdi, A.S. (2009). Optimum step stress accelerated life testing for Lomax distibution, Journal of Applied Sciences Research 5, 2153-2164.

13. Lemonte, A. J. and Cordeiro, G. M. (2013). An extended Lomax distribution. Statistics, 47(4), 800-816.

14. Lomax, K.S. (1954). Business failures: Another example of the analysis of failure data, Journal of the American Statistical Association 49, 847-852.

15. Murthy, D.N.P. Xie, M. and Jiang, R. (2004). Weibull Models, Wiley.

16. Silva, F. S., Percontini, A., de Brito, E., Ramos, M. W., Venancio, R. and Cordeiro, G. M. (2017). The Odd Lindley-G Family of Distributions. Austrian Journal of Statistics, 46 (1), 65-87.

17. Tahir, M. H., Cordeiro, G. M., Mansoor, M. and Zubair, M. (2015). The Weibull-Lomax distribution: properties and applications. Hacettepe Journal of Mathematics and Statistics, 44(2), 461-480.

18. Yousof, H. M., Afify, A. Z., Hamedani, G. G. and Aryal, G. (2017). The Burr X generator of distributions for lifetime data. Journal of Statistical Theory and Applications, 16, 288-305.

19. Yousof, H. M., Ahsanullah, M. and Khalil, M. G. (2019). A New Zero-Truncated Version of the Poisson Burr XII Distribution: Characterizations and Properties. Journal of Statistical Theory and Applications, 18(1), 1-10.

20. Yousof, H. M., Rasekhi, M., Alizadeh, M. and Hamedani G. G. (2018). The MarshallOlkin exponentiated generalized G family of distributions : properties, applications and characterizations. Journal of Nonlinear Sciences and Applications, forthcoming. 